\title{
乌s \\ Vertical orbit excursion fixed field alternating gradient accelerators
}

\author{
Stephen Brooks* \\ RAL, Chilton, OX11 0QX, United Kingdom \\ (Received 30 April 2013; published 6 August 2013)
}

\begin{abstract}
Fixed field alternating gradient (FFAG) accelerators with vertical orbit excursion (VFFAGs) provide a promising alternative design for rings with fixed-field superconducting magnets. They have a vertical magnetic field component that increases with height in the vertical aperture, yielding a skew quadrupole focusing structure. Scaling-type VFFAGs are found with fixed tunes and no intrinsic limitation on momentum range. This paper presents the first multiparticle tracking of such machines. Proton driver rings to accelerate the $800 \mathrm{MeV}$ beam from the ISIS synchrotron are presented, in terms of both magnet field geometry and longitudinal behavior during acceleration with space charge. The $12 \mathrm{GeV}$ ring produces an output power of at least 2.18 MW. Possible applications of VFFAGs to waste transmutation, hadron therapy, and energy-recovery electron accelerators are also discussed.
\end{abstract}

DOI: 10.1103/PhysRevSTAB.16.084001

PACS numbers: 41.75.Lx, 41.85.Ja, 41.20.-q

\section{VFFAG PRINCIPLE}

In what follows, an FFAG is an accelerator with fixed magnetic fields but alternating gradient focusing over a range of energies; a VFFAG is such an accelerator where the vertical position of the beam changes with energy.

If the closed orbit of a ring accelerator lies in a horizontal plane (constant $y$ ), the magnetic field along it must satisfy $\oint B_{y} \mathrm{~d} s=2 \pi p / q$ in order for the orbit to close, where $s$ is path length. This can be satisfied by many magnetic fields, some of which permit vertical orbit excursion. If $B_{y}$ increases with $y$, the closed orbit will move in the $+y$ direction (upwards) as $p$ increases, so that $\oint B_{y} \mathrm{~d} s$ remains proportional to beam momentum. In what follows, a local Cartesian frame is used with the beam moving in the $+z$ direction and $x$ being the horizontal direction perpendicular to $y$ and $z$.

It is a misconception to think that the closed orbit always moves to larger radii as momentum increases in a fixed field machine. In fact the beam will adiabatically track any closed orbit provided that it has locally stable optics. In the vertical excursion case, increased beam rigidity in a given $B_{y}$ field initially moves particles horizontally outwards into regions where the vertical field gradient implies (via $\nabla \cdot \mathbf{B}=0$ ) that there is a $B_{x}$ component, which then pushes the particles upwards as required.

Figure 1 shows an example of the motion of a beam in such an accelerator with increasing energy, where adiabatic shrinkage of the beam is also visible.

\footnotetext{
*stephen.brooks@stfc.ac.uk
}

Published by the American Physical Society under the terms of the Creative Commons Attribution 3.0 License. Further distribution of this work must maintain attribution to the author(s) and the published article's title, journal citation, and DOI.

\section{A. Linear optics of scaling VFFAGs}

This paper concentrates on what could be termed the scaling vertical orbit excursion field by analogy with (horizontal) scaling FFAGs. The magnetic fields in scaling machines are derived from a symmetry principle. If a closed orbit of length $\ell$ is scaled by a factor $a$ in size (and possibly translated or rotated) while magnetic fields on that orbit are scaled by $b$, then the beam momentum $p \propto$ $B \ell$ must scale by $a b$. Scaling FFAGs use a group of transformations that scale about the ring center with $b=a^{k}$

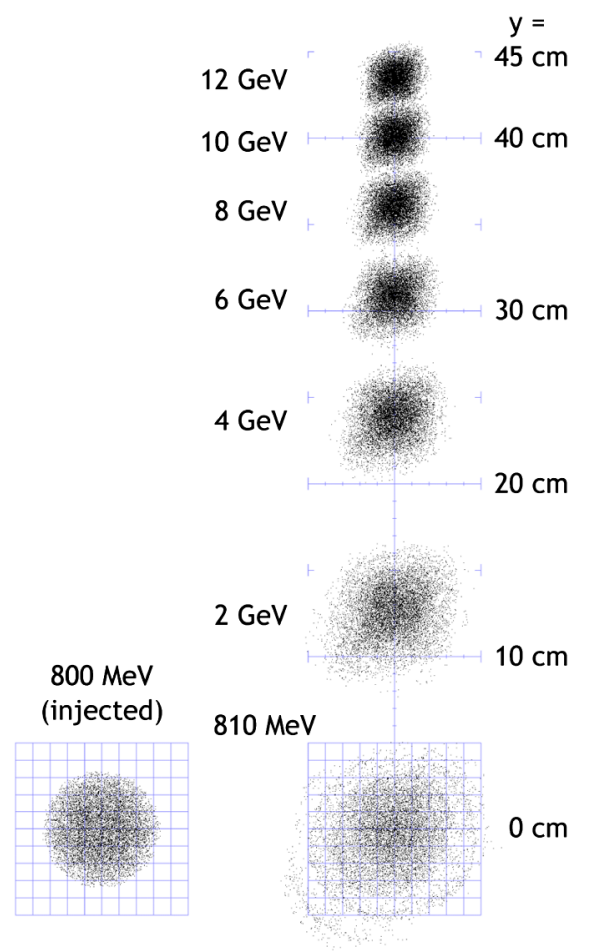

FIG. 1. Vertical movement of a proton beam accelerated in a scaling VFFAG with $k=5 \mathrm{~m}^{-1}$ [3]. 
$[1,2]$ for some field index $k$. Scaling VFFAGs as defined here use a group of translations for which $a=1$ but $b=$ $\mathrm{e}^{k \Delta y}$ for vertical orbit offset $\Delta y . k$ must be constant for the entire ring to satisfy the scaling law

$$
y \mapsto y+\Delta y, \quad(p, \mathbf{B}) \mapsto(p, \mathbf{B}) \mathrm{e}^{k \Delta y},
$$

which ensures the geometry of all particle trajectories, including those off the closed orbit, are preserved during acceleration: translating upwards as $p$ is increased.

Thus, the field within the body of a scaling VFFAG magnet is given by $B_{y}=B_{0} \mathrm{e}^{k y}$ on the $x=0$ closed orbit surface and the beam shifts to height $y=\frac{1}{k} \ln p / p_{\text {inj }}$ as momentum $p$ increases. This paper assumes the orbit curvature within each magnet is small so field expansions treating $x=0$ as a flat "midplane" are valid. The injection orbit is defined to be at $y=0$ and the current windings lie on the $\pm x$ sides of a vertical gap (see Figs. 3 and 5). At injection, the magnet body has bending field $B_{0}$ and skew gradient $B_{0} k$ (as well as higher multipoles of strength proportional to $B_{0} k^{n \geq 2}$ ), so without edge effects $B_{0}$ must alternate in sign to provide alternating gradient focusing. Optics of a ring with such magnets without edge effects are considered in [3], including scans of the lattice stability regions to find which have good dynamic aperture in spite of the nonlinear fields.

As in scaling FFAGs, this magnetic field is a combined function, with a dipole and gradient (and all higher multipoles) superimposed. For small distances from the origin, the field is to first order

$$
B_{y}=B_{0}+B_{0} k y+\cdots, \quad B_{x}=-B_{0} k x+\cdots,
$$

which produces skew focusing optics as shown in Fig. 2. It is useful to define skew transverse axes,

$$
u=(x+y) / \sqrt{2}, \quad v=(y-x) / \sqrt{2},
$$

which are rotated by $45^{\circ}$. Using these, the usual expression for a quadrupole field reappears:

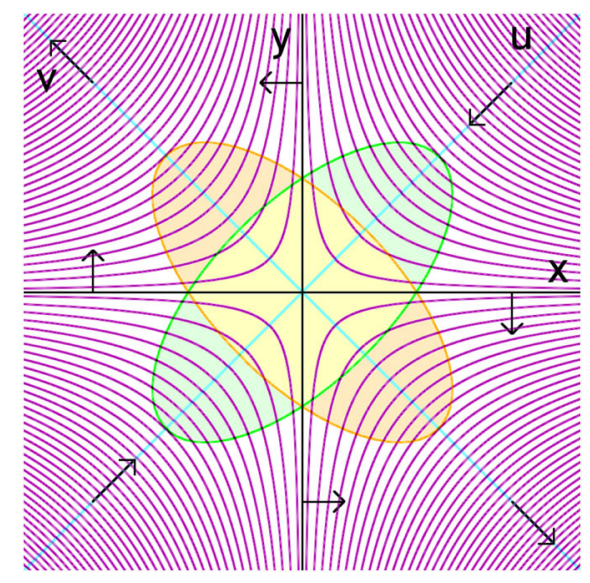

FIG. 2. Skew quadrupole magnetic field (purple lines) and direction of forces (arrows) exerted on the beam, which in a skew FODO channel oscillates in shape between the two ellipses shown. The new skew axes $u, v$ are labeled.

$$
\begin{aligned}
B_{u} & =\left(B_{x}+B_{y}\right) / \sqrt{2}=B_{0} / \sqrt{2}+B_{0} k(y-x) / \sqrt{2} \\
& =B_{0} / \sqrt{2}+B_{0} k v, \\
B_{v} & =\left(B_{y}-B_{x}\right) / \sqrt{2}=B_{0} / \sqrt{2}+B_{0} k(x+y) / \sqrt{2} \\
& =B_{0} / \sqrt{2}+B_{0} k u .
\end{aligned}
$$

Another important feature shared with scaling FFAGs is that optics are identical at each momentum, so tunes stay constant, which is important for proton machines with many turns or significant space charge. Scaling VFFAGs have other interesting properties, such as constant dispersions $D_{x}=0$ and $D_{y}=\frac{1}{k}$ and a constant orbit length that makes $\gamma_{t r}=\infty$.

\section{B. Combination with existing FFAG types}

For most horizontal orbit excursion FFAGs, there is a VFFAG with the same focusing structure, with similar orbit separation and field gradients, except the separation becomes vertical and the gradients skew. The VFFAG may also be combined with fixed or variable-frequency rf acceleration schemes.

In scaling VFFAGs, field gradient is proportional to the bending field, which has the unfortunate consequence of requiring reverse bends in all alternating gradient lattices. Having negative $B_{0}$ for some magnets in this way increases machine circumference for a given field by $\sim 5$ times, similar to the circumference factor $[1,4]$ in horizontal scaling FFAGs. The parameter space for scaling focusoff-defocus-off (FODO) and triplet VFFAG lattices is plotted in [3], showing most of the stable region stays close to the $F=D$ line where net bending is zero. This can be mitigated somewhat by using edge angles on the magnets for the alternating focusing and the interior field for bending, permitting a single magnet per lattice cell. This technique is the equivalent of the "spiral sector" scaling FFAG and is used in the proton driver machine presented in Secs. III and IV; it is also commonly used in cyclotrons.

Nonscaling VFFAGs are also possible but as with horizontal nonscaling FFAGs, tunes may no longer be fixed and momentum range may be limited. Additionally, the closed orbits will not necessarily stack vertically on top of each other due to the different horizontal bending experienced by different momenta in each magnet, meaning the orbit excursion could sweep out a generalized curved surface. Linear-field nonscaling VFFAGs, suggested by [5], can be constructed using only offset skew quadrupoles, though their beam dynamics is at present completely unexplored.

Adding a synchrotron-like rf system to a VFFAG permits acceleration of bunches of any energy, with the $\mathrm{rf}$ frequency tracking the ring revolution frequency, provided the rf has the appropriate aperture slot for the beam orbit excursion. Fixed-frequency rf can also be considered for ultrarelativistic beams, since the VFFAG closed orbit circumference does not change very much with energy and is constant in scaling VFFAGs, making the ring nearly isochronous as $v \rightarrow c$. This could enable cyclotron-like $\mathrm{cw}$ operation for some machines. 


\section{Historical papers}

The earliest mention of the VFFAG principle the author is aware of is Ohkawa [6] in 1955, who suggested a scaling VFFAG for electrons with fixed-frequency rf, calling it an FFAG cyclotron with fixed orbit length. Leleux et al. [7] in 1959 again found the exponential field configuration and analyzed its linear dynamics and stability in their report. They call it a "helicoidal FFAG" after the helical progression of the orbit upwards in the ring as it is accelerated. Teichmann [8] in 1962 had continued developing Ohkawa's idea in order to achieve "complete isochronism," that is a fixed-tune isochronous FFAG, and presents an interesting figure showing that a deviation from exact vertical orbit excursion can make the orbits of even subrelativistic particles exactly isochronous. This will be revisited briefly in Sec. VB.

The author developed most of these ideas independently starting in 2009, discovering this literature later with the help of colleagues. New developments in the intervening half century include the widespread use of computerized multiparticle tracking and superconducting magnets in accelerators, both of which will be discussed in this paper.

\section{MAGNETIC FIELD MODELS}

If conductors rather than iron dominate, as is the case for high-field superconducting or bare coil magnets, producing a vertical magnetic field in a vertical slot aperture can be
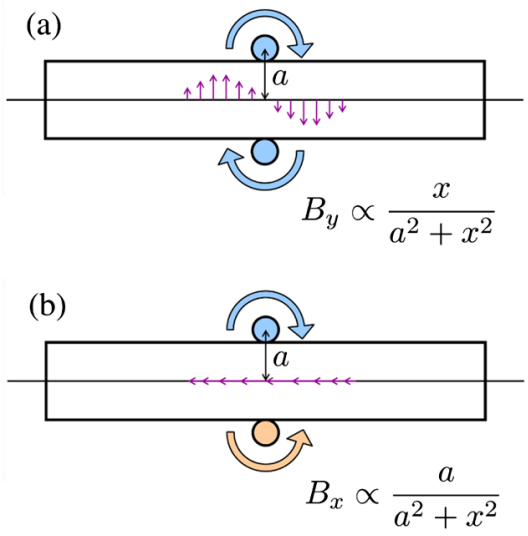

(c)

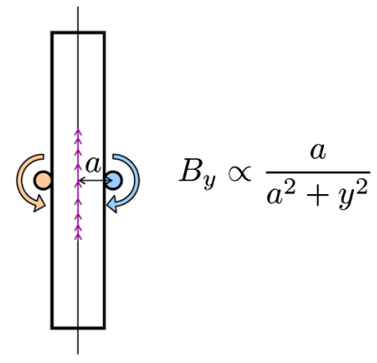

FIG. 3. Pairs of conductors with currents perpendicular to the page that create: (a) a vertical field in a horizontal aperture; (b) a horizontal field in a horizontal aperture; (c) a vertical field in a vertical aperture. easier than in the horizontal slot required by conventional FFAGs. Figure 3(a) shows that conductors parallel to the beam direction placed symmetrically above and below a horizontal slot must actually cancel each other at their midpoint in order to produce a pure $B_{y}$ field on the midplane. The field is produced some distance to the side of the coils; normal conducting synchrotron dipoles resemble split solenoids on their side for this reason, with the coil at the periphery of the beam region (and field enhanced by iron).

Reversing the current in one of the conductors so that the fields are additive on the midplane produces more field closer to the sources but now the field is horizontal. This problem can be turned into an advantage by rotating the whole magnet so that both the field and aperture slot are vertical: this is the VFFAG situation. It is favorable for conductor-dominated magnets because the field is produced from conductors closer to the beam position and unfavorable for iron-dominated magnets that would make the field perpendicular to the slot sides rather than parallel.

\section{A. Analytic exponential model (2D)}

For the field in a long magnet with no variation in $z$, Maxwell's equations in free space reduce to

$$
\partial_{x} B_{x}+\partial_{y} B_{y}=0, \quad \partial_{x} B_{y}-\partial_{y} B_{x}=0,
$$

and $B_{z}$ constant, assumed here to be zero. The solution to these equations that gives the scaling VFFAG field $B_{y}=$ $B_{0} \mathrm{e}^{k y}$ and $B_{x}=0$ on the midplane $x=0$ is

$$
B_{y}=B_{0} \mathrm{e}^{k y} \cos k x, \quad B_{x}=-B_{0} \mathrm{e}^{k y} \sin k x .
$$

This field is plotted in Fig. 4: its strength increases exponentially with $y$ everywhere, satisfying the scaling law, and the field vector rotates with $x$ off the midplane.

The 2D free space Maxwell equations are exactly the conditions for the complex function $f(x+\mathrm{i} y)=B_{y}+\mathrm{i} B_{x}$ to be holomorphic. In this case the solution corresponds to $f(z)=B_{0} \mathrm{e}^{-i k z}$, the complex exponential explaining the appearance of sin and cos in the off-plane field.

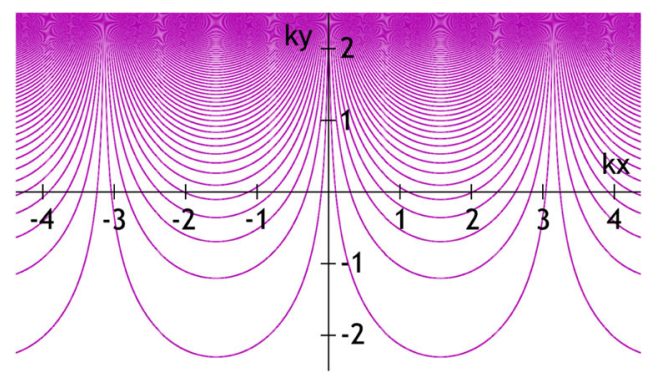

FIG. 4. Transverse field lines of the scaling VFFAG field in a long magnet derived analytically from the complex exponential function. 


\section{B. Block conductors model (2D)}

To see if the exponential field could be produced by a practical arrangement of superconductors, a 2D model made of rectangular block coils carrying $250 \mathrm{~A} / \mathrm{mm}^{2}$ was optimized to produce the $k=5 \mathrm{~m}^{-1}$ field from $0.5-4.41 \mathrm{~T}$ used in [3]. Figure 5 shows the result, with the coils on the $+x$ side having opposite polarity to those on the $-x$ side. The exponential field was produced well with small $(<1 \%)$ field errors, though in a real magnet the design would be optimized more finely. Because the machine in question had a large energy range of $0.8-12 \mathrm{GeV}$, the design took advantage of the relativistic beam shrinkage by bringing the coils for the highest energies closer to the midplane.

It is interesting to note that for smaller beams, the magnetized volume could be made very small by narrowing the gap between the conductors into a thin layer. As the conductors have opposite polarity, forces on the coils are away from the midplane, so an external support structure in compression should keep them in place.

\section{Series solution with end fields (3D)}

A three-dimensional field model must also include the ends of the magnet. Maxwell's equations for free space will extrapolate the field for the interior of the magnet once it is specified on a plane: here the $x=0$ midplane is chosen. To represent magnets with edge angles, as found in spiral scaling VFFAGs, the parameter $\tau=\tan \theta_{\text {edge }}$ is introduced, along with a coordinate $\zeta=z-\tau y$ so that the magnet corresponds to the region $0 \leq \zeta \leq L_{\text {mag }}$ for all $y$. Field falloff is determined by a function $f(\zeta)$ that approaches 1 in the magnet body and 0 outside. Naively one wants a midplane field $B_{y}=B_{0} \mathrm{e}^{k y} f(\zeta)$ but to obey Maxwell's
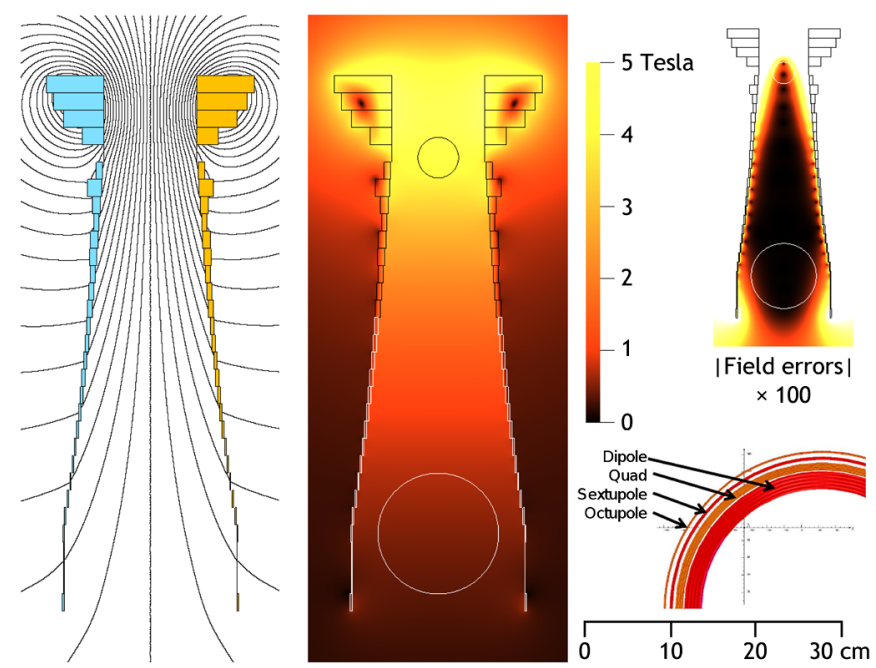

FIG. 5. 2D scaling VFFAG magnet design using block coils: left: field lines and coils; center: field strength and proton beam sizes at 0.8 and $12 \mathrm{GeV}$; right: field errors and the size of the superconducting windings in the PAMELA [22] scaling FFAG, to scale.

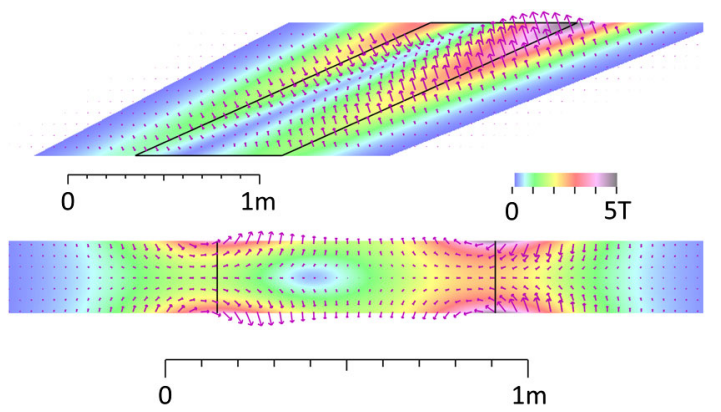

FIG. 6. Cross section of the $0.8-5 \mathrm{GeV}$ proton driver ring magnet's field in $Z Y$ (top) and $Z X$ (bottom) planes. It has $k=$ $2.01 \mathrm{~m}^{-1}, \tau=2.23$, and $B_{0}=0.5 \mathrm{~T}$.

equation $(\nabla \times \mathbf{B})_{x}=0$, this has to be modified to $\left(B_{y}, B_{z}\right)=B_{0} \mathrm{e}^{k y}\left(f(\zeta)-\frac{\tau}{k} f^{\prime}(\zeta), \frac{1}{k} f^{\prime}(\zeta)\right)$. The note [9] derives this formula and a Taylor series extrapolation of the field for $x \neq 0$. The author wrote a program in $\mathrm{C}$ to calculate the field at any point by summing this Taylor series. For edge angles, $z \mapsto z+\tau \Delta y$ is added to the VFFAG scaling law to keep $\zeta$ constant (more accurately, this is a rotation of $\tau \Delta y / R$ about the ring center).

An example of the resulting field is plotted in Fig. 6. The fringe field at the entrance to the magnet has opposite sign to that at the exit, providing alternating gradient focusing without changing the sign of $B_{0}$. Note that symmetry about the $Y Z$ plane forbids conventional quadrupole fields, meaning all focusing is skew apart from the $B_{z}$ component with focusing like a solenoid.

\section{Winding configuration}

As with the 2D magnet, the conductors will be parallel to the beam ( $z$ axis) in the body of the magnet, running in opposite directions each side of the $x=0$ midplane, but the circuits must close somehow at the ends. This should be achieved in practice by the conductors running to the top of the magnet along the $\zeta=0$ and $\zeta=L$ lines and then looping over to the other side of the midplane. In fact, the accumulation of conductors running up the ends of the magnet is what produces the end fields, since Maxwell's equations implicitly require closed circuits with $\nabla \cdot \mathbf{J}=0$. A detailed 3D model of the winding configuration is a topic for further study.

\section{Field enhancement factor}

As can be seen in Fig. 6, the largest fields are present in the magnet edges and off plane. The field enhancement factor

$$
F_{\text {enh }}=\frac{\max _{z}|\mathbf{B}(x, y, z)|}{B_{0} \mathrm{e}^{k y}}=\frac{B_{\text {fringe }}}{B_{\text {body }}}
$$

is plotted in Fig. 7 (at $y=0$, though by the scaling law it is the same at all $y$ ). 


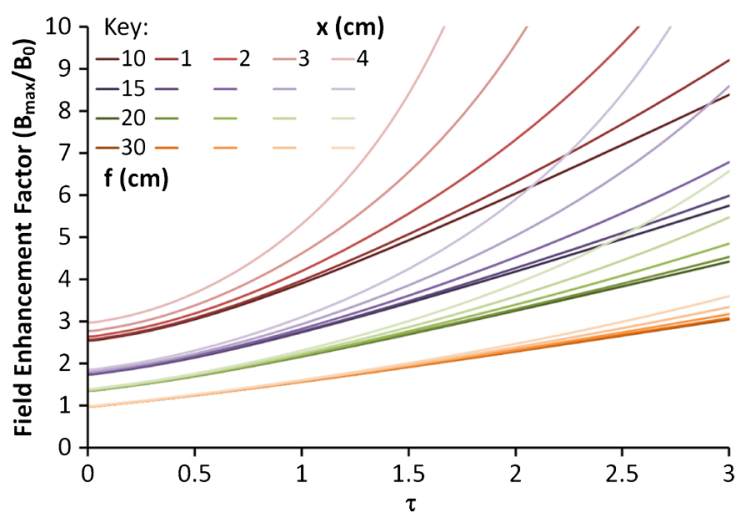

FIG. 7. Field enhancements as a function of $\tau$, fringe length $(f)$, and distance from midplane $(x)$ from 0 to $4 \mathrm{~cm}$, in the 3 or $5 \mathrm{GeV}$ magnet design with $k=2.05 \mathrm{~m}^{-1}$.

Enhancement increases with $\tau$ but is ameliorated by increasing fringe length; it also increases extremely rapidly with $x$ for small fringe lengths. However, it decreases with increasing $k$ because higher $k$ magnets actually have weaker fringe fields.

In ring design, this number fills a similar role to the circumference factor of scaling FFAGs: it is the amount a theoretical constant bending field must be multiplied by to find the real maximum field strength in a ring of fixed size and magnet filling factor.

\section{Circumference factor comparison}

Symon $[1,4]$ defined "circumference factor" to be the length of the top-energy closed orbit divided by the circumference of a circle with the maximum curvature found anywhere on the orbit, which is equivalent to $C=|\mathbf{B}|_{\max } /\left\langle B_{y}\right\rangle$ where $\langle\cdot\rangle$ denotes average around the orbit. For spiral scaling VFFAGs with a singlet lattice, $C=F_{\text {enh }} / P$ where $P$ is the magnet packing factor. For the proton driver lattices considered in the next section, $F_{\text {enh }}=2.6-2.7$ and $P^{-1}=$ 4.3-5.3, giving $C=11.2-14.4$. For a synchrotron, $C=$ $P^{-1}$, determined only by the drift space requirements. The original MURA note considered FFAGs entirely filled with magnets $(P=1)$ when it quoted values of $C=5-6$ for scaling FFAGs, so $C P$ may be a better metric for comparing lattice bending efficiency independently of packing factor. This is just equal to $F_{\text {enh }}$ for the spiral VFFAGs and is somewhat lower than the values for sector scaling FFAGs with reverse bends, even though $B_{y}$ goes locally negative in the spiral VFFAG magnet's exit end field.

\section{PROTON DRIVER TRANSVERSE DYNAMICS}

Parameters were sought for fixed-field rings to boost the energy of the two ISIS [10] proton bunches from $800 \mathrm{MeV}$, following the outline rf approach in [11]. Lattice cells containing a single VFFAG magnet and a reasonably long drift space, with enough dynamic aperture for the $150 \mathrm{~mm}$ mrad geometric emittance proton beam are given in Table I.
TABLE I. Transverse parameters for VFFAG rings.

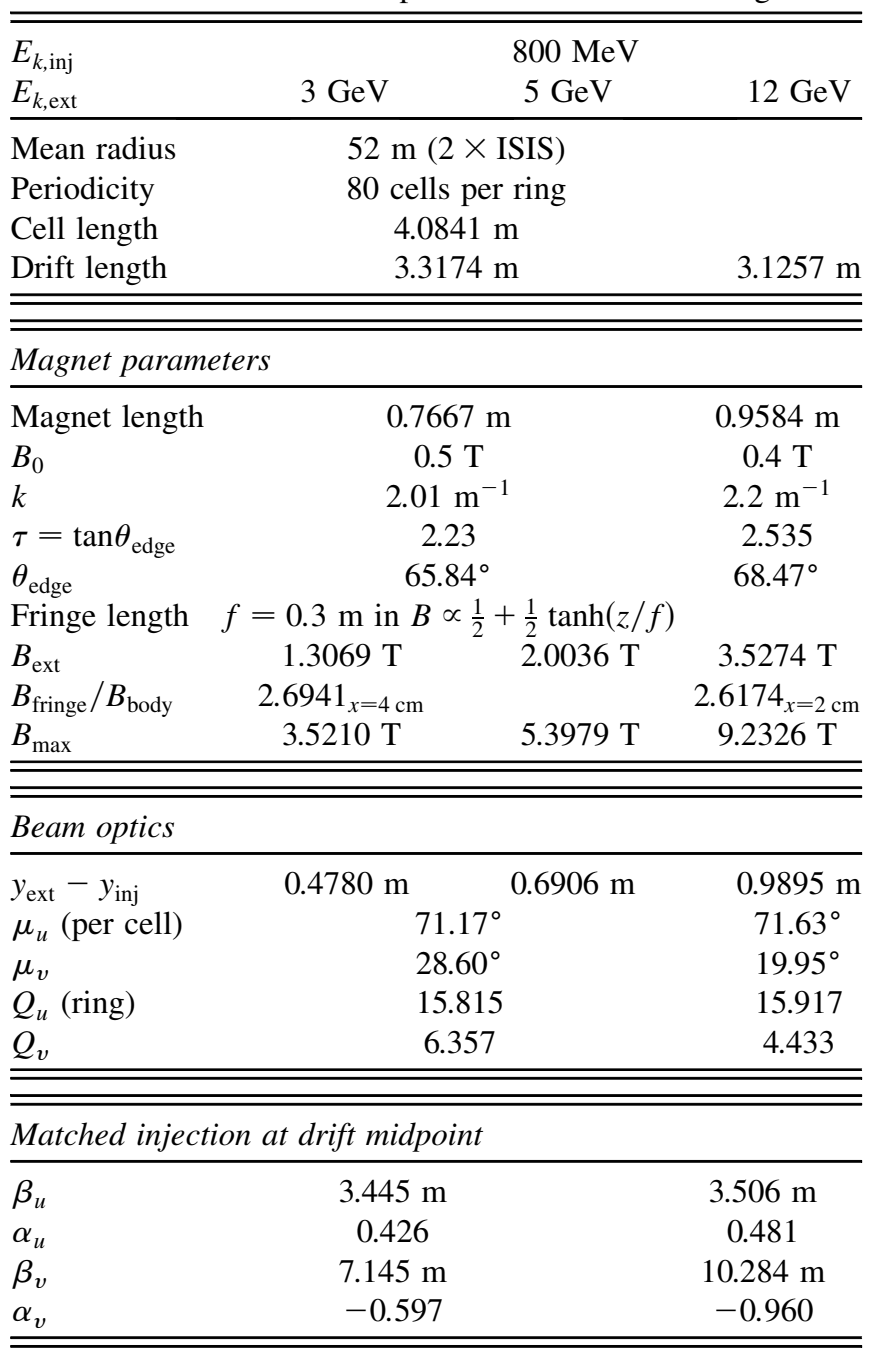

The three-dimensional layout of such a machine is shown in Fig. 8.

The beam power will increase in proportion to energy, so options are provided for neutron production at $3 \mathrm{GeV}$, highpower exotics production at $12 \mathrm{GeV}$ and a "compromise" energy of $5 \mathrm{GeV}$, which provides more power for neutrons but perhaps less efficiency. With the mean current $208 \mu \mathrm{A}$ presently achievable in ISIS, these would have beam powers of $0.6,2.5$, and $1.0 \mathrm{MW}$, respectively, at $50 \mathrm{~Hz}$.

The $12 \mathrm{GeV}$ ring, the most aggressive design, with applications to neutrino factories and muon colliders, needed a slightly longer magnet to lower the peak field, which in turn required larger edge angles. The field enhancement was evaluated at $x=2 \mathrm{~cm}$ and not $4 \mathrm{~cm}$ to

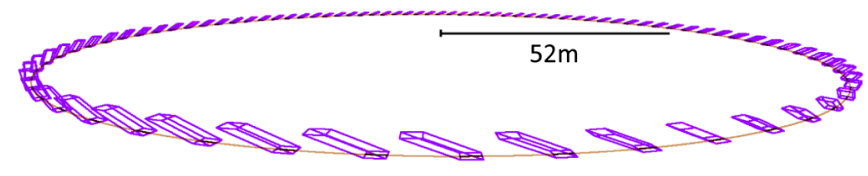

FIG. 8. Perspective view of the $12 \mathrm{GeV}$ ring. 

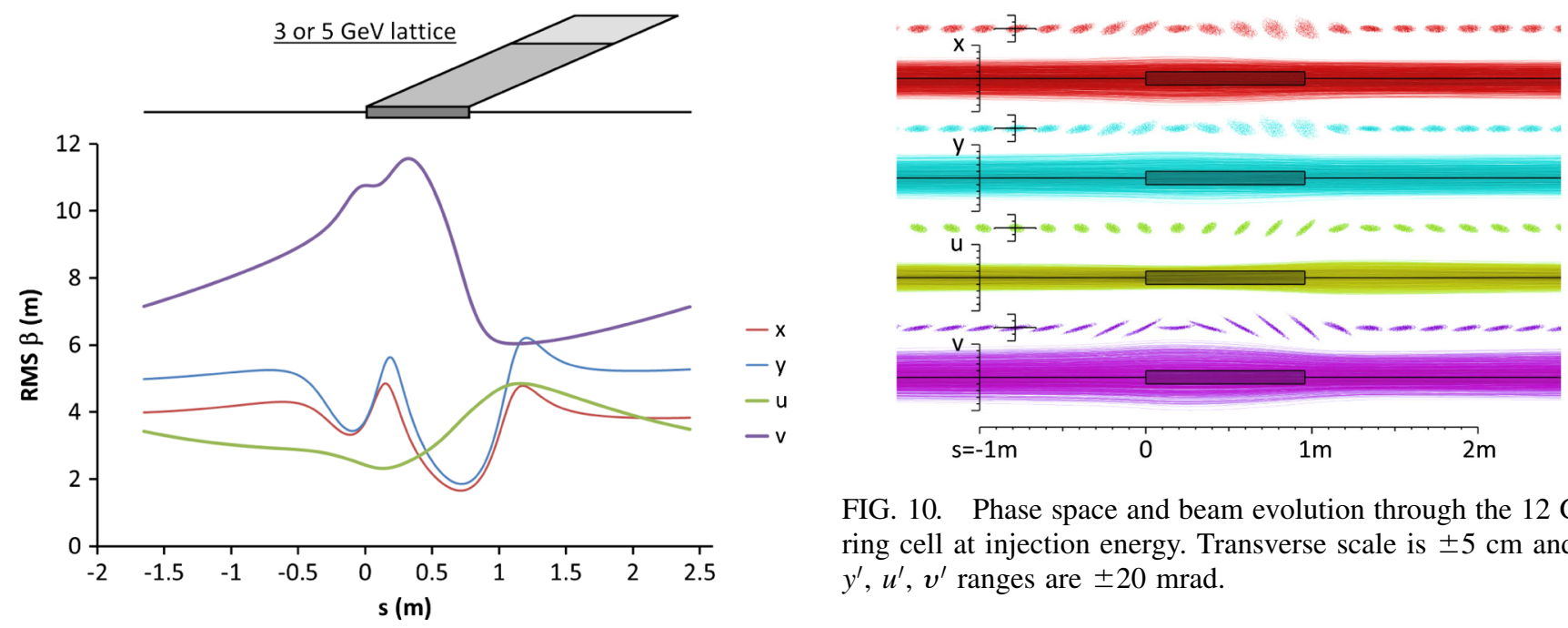

FIG. 10. Phase space and beam evolution through the $12 \mathrm{GeV}$ ring cell at injection energy. Transverse scale is $\pm 5 \mathrm{~cm}$ and $x^{\prime}$, $y^{\prime}, u^{\prime}, v^{\prime}$ ranges are $\pm 20 \mathrm{mrad}$.

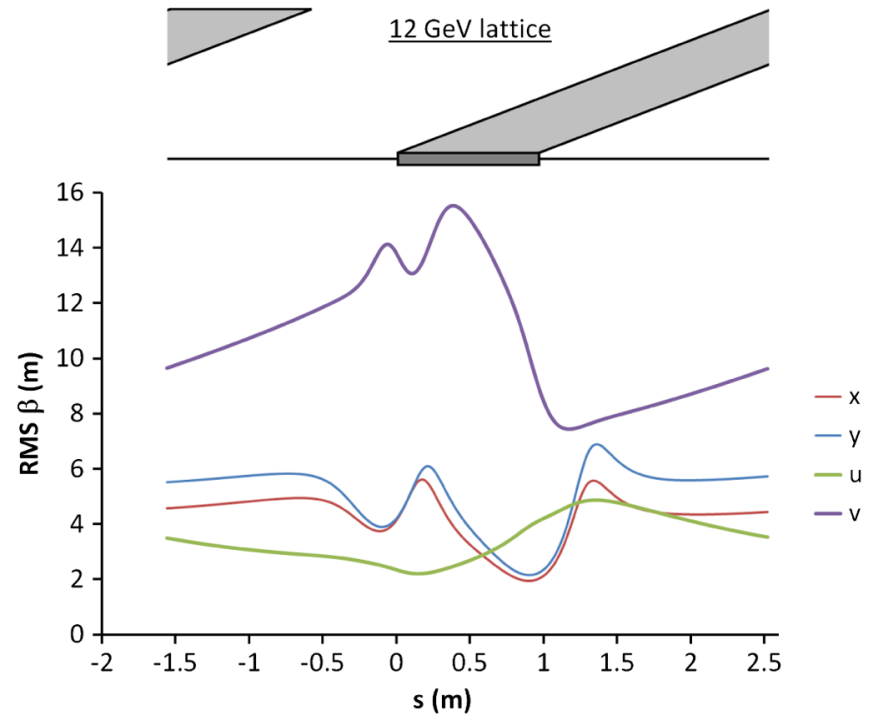

FIG. 9. Root-mean square beta functions $\left(\beta_{x}^{\mathrm{rms}}=\sigma_{x}^{2} / \epsilon_{x}^{\mathrm{rms}}\right)$ of the beam distribution at injection in the two lattices, in nonskew and skew coordinates. In the schematic cell diagrams, magnet size is to scale in $z$ and $y$.

account for adiabatic shrinkage of the beam once accelerated to $12 \mathrm{GeV}$.

In terms of skew coordinates $u$ and $v$, the lattice beta functions shown in Fig. 9 are overall doublet-like but with some features in the end fields. The $x$ and $y$ optics are highly coupled so do not behave like normal beta functions. The cell and machine tunes in Table I are also given in terms of skew axes, although since the weak focusing in $x$ from the machine curvature couples even these, the values are eigentunes that have been labeled as primarily $u$ or $v$. Figure 10 shows how the phase spaces vary through the magnet, with some distortion of the matched shape, particularly in the $\left(v, v^{\prime}\right)$ plane due to nonlinearity in the magnetic field.

Transverse multiparticle tracking without space charge was performed using a 2nd order drift-kick tracking

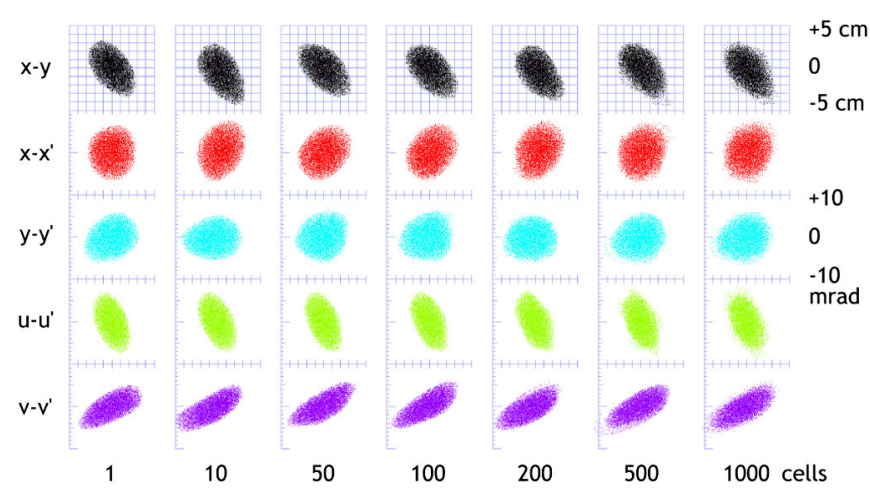

FIG. 11. Tracking through $\sim 12$ turns of the $12 \mathrm{GeV}$ ring with matched beams (no space charge). Optics stable apart from signs of a 5 th order resonance in the $\left(u, u^{\prime}\right)$ plane, since $\mu_{u} \simeq 72^{\circ}$.

method, which is symplectic apart from large-angle terms (e.g. spherical aberration) and 200 steps per cell. The results for a 10000 particle distribution are shown in Fig. 11.

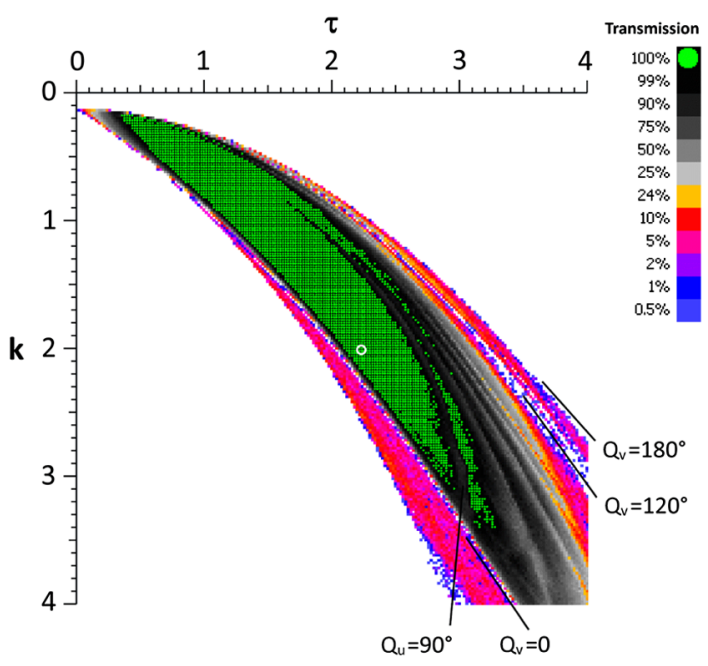

FIG. 12. Proton beam transmission as a function of $\tau$ and $k$, with the 3 or $5 \mathrm{GeV}$ ring design circled. Lines of increased loss correspond to cell tune resonances (labeled). 


\section{A. Dynamic aperture parameter scan}

The ring designs were found as combinations of six parameters $\left(B_{0}, k, \tau, f, L_{\text {mag }}, L_{\text {drift }}\right)$, the last two being dictated by the integer rf harmonic number (ring circumference) and superperiodicity (cell length) together with $B_{0}$, which gives the magnet fill factor. The main focusing parameters $k$ and $\tau$ were scanned over, producing plots like Fig. 12. For each square, 250 protons from a $150 \mathrm{~mm}$ mrad waterbag beam were tracked for 250 cells and removed if $r>10 \mathrm{~cm}$. Squares are colored according to the percentage that survive, showing areas of good dynamic aperture.

\section{B. Transverse intensity issues}

Since the ring tune is 80 times the cell tune, a fine-tuning stage is needed to steer the fractional parts of the ring tunes away from resonances. For the 3 or $5 \mathrm{GeV}$ ring,

$$
\frac{\partial Q_{u, v}}{\partial k}=\left[\begin{array}{c}
-8.49 \\
-94.46
\end{array}\right] \text { and } \frac{\partial Q_{u, v}}{\partial \tau}=\left[\begin{array}{c}
39.92 \\
119.82
\end{array}\right] \text {, }
$$

which are linearly independent enough to find any desired fractional ring tunes without major deterioration of the optics. This fine-tuning will also have to be done on the real machine, using trim coils producing fields proportional to $\partial \mathbf{B} / \partial k$ and $\partial \mathbf{B} / \partial \tau$.

The rapid variation of $Q_{v}$ arises because the cell tune in $v$ is quite close to zero. This is problematic since $Q_{v}$ also varies rapidly in response to space charge forces, making the tune depressions of these rings roughly $\Delta Q_{s c, u}=-0.2$ and $\Delta Q_{s c, v}=-0.4$ at injection. This could be improved by finding rings with more balanced tunes or larger mean beta functions, though maybe at the expense of shorter drift spaces or a larger circumference.

\section{PROTON DRIVER LONGITUDINAL DYNAMICS}

The line charge density $\rho_{1 \mathrm{D}}$ along the beam determines the level of transverse as well as longitudinal space charge, so it is important to do realistic simulations of the longitudinal bunch shape including space charge. The simulation code written for this uses three transformations on the particle $(\Delta t, \Delta E)$ coordinates: a drift, an rf kick, and a space charge kick; doing these once per turn provides sufficient accuracy. The drift affects $\Delta t$ depending on the ring circumference function $C\left(E_{k}, t\right)$. For a synchrotron, the absolute time $t$ determines the machine magnet reference momentum $p_{0}$, then the particle's $E_{k}$ gives its $\Delta p / p$, so to first order $C=C_{0}\left(1+\alpha_{0} \Delta p / p\right)$ where $\alpha_{0}$ is the momentum compaction ( 0.0392 for ISIS). Fixed field magnets have no $t$ dependence and for a scaling VFFAG the circumference does not change with closed orbit momentum either, so $C$ is constant. The rf and space charge kicks affect $\Delta E$ only, so when done separately from the drift, phase space area is preserved.

Radio-frequency voltages applied to a proton per turn are defined as $-V_{0} \sin \left(\phi_{0}+2 \pi f \Delta t\right)$, with $V_{0}(t)$, $\phi_{0}(t)$

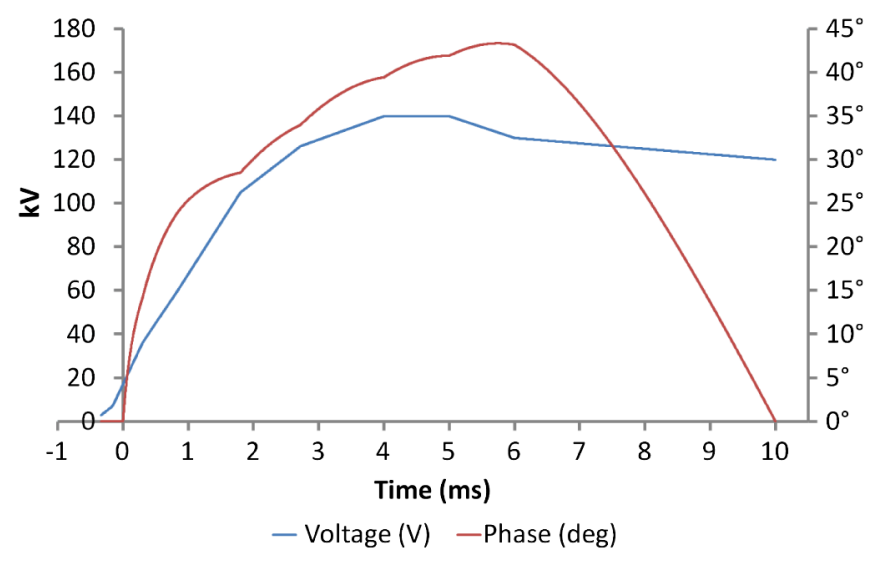

FIG. 13. ISIS first harmonic rf program.

specified by the user. Space charge is calculated using the derivative of the line density, which is Gaussian smoothed to reduce statistical noise. Provided $\frac{\mathrm{d}^{2} \rho_{\mathrm{DD}}}{\mathrm{d} z^{2}}$ is small, the voltage per turn from space charge is

$$
V_{\mathrm{sc}}=-C\left\langle E_{z, \mathrm{sc}}\right\rangle=\frac{C}{4 \pi \epsilon_{0}} \frac{g}{\gamma^{2}} \frac{\mathrm{d} \rho_{1 \mathrm{D}}}{\mathrm{d} z},
$$

where $g$ is a shielding factor from the shape of the beam and the conducting beam pipe [12]. ISIS has $g=1.546$ in a mostly round beam pipe, while the VFFAG has $g=1.914$ for a skew Gaussian beam between two vertical conducting plates at $x= \pm 4 \mathrm{~cm}$, calculated using the method in [13].

To obtain an input distribution for the VFFAG (and as a check), 1D simulations were first run on ISIS starting from the linac injection at $70.44 \mathrm{MeV}$. The voltage and phase functions [14] for this are shown in Fig. 13 and the results in Fig. 14 agree with the current models and observed transmission values of the machine in this mode.

\section{A. Longitudinal intensity effects}

Figure 14 shows a number of parameters that could limit machine intensity. The bunching factor is defined as $B=$ $\left\langle\rho_{1 \mathrm{D}}\right\rangle / \rho_{1 \mathrm{D}}^{\text {peak }}$ with smaller values being worse for space

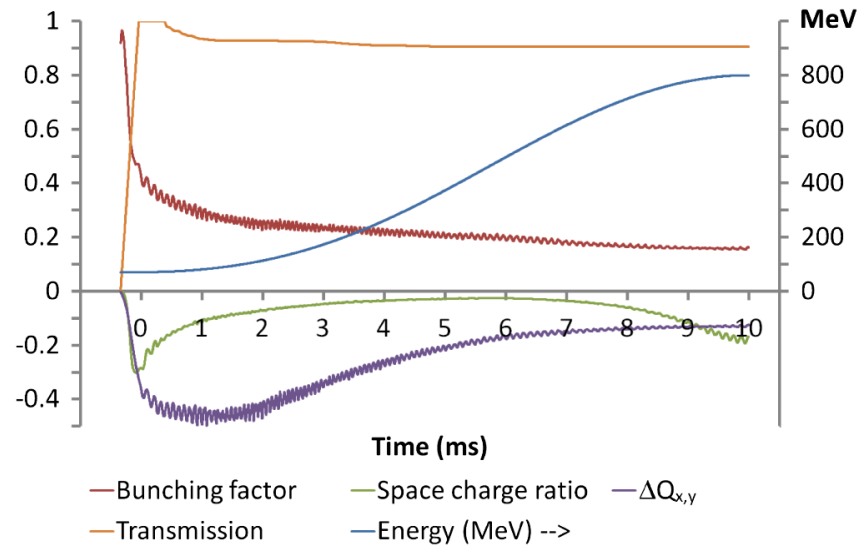

FIG. 14. Bunching factor, transmission, and intensitydependent effects in the ISIS first harmonic simulation. 
TABLE II. Longitudinal parameters for the $12 \mathrm{GeV}$ VFFAG. Peak voltage per turn and phase are linearly interpolated from the times given.

\begin{tabular}{lcc}
\hline \hline rf harmonic & \multicolumn{2}{c}{$h=8$} \\
rf frequency & \multicolumn{1}{c}{$\begin{array}{c}\text { C.179-7.321 MHz } \\
\text { Cycle duration } \\
\text { Repetition rate }\end{array}$} & \multicolumn{2}{c}{$50 \mathrm{~ms}$} \\
\hline Time $(\mathrm{ms})$ & Voltage $(\mathrm{kV})$ & Phase \\
\hline 0 & 150 & $10^{\circ}$ \\
1 & 250 & $20^{\circ}$ \\
2 & 350 & $25^{\circ}$ \\
2.5 & 525 & $30^{\circ}$ \\
3 & 800 & $35^{\circ}$ \\
4 & 1000 & $40^{\circ}$ \\
10 & 1000 & $55^{\circ}$ \\
18.41 (extract) & 1000 & $59.21^{\circ}$ \\
20 & 1000 & $60^{\circ}$ \\
\hline \hline
\end{tabular}

charge. The "space charge ratio" is defined as an average of $V_{\mathrm{sc}} / V_{\mathrm{rf}}$ over the beam, roughly equivalent to $\Delta Q_{s} / Q_{s}$ in terms of the synchrotron tune. It has been shown using the Boussard criterion [15] that this must be greater than -0.4 to avoid microwave instabilities. To avoid dividing by zero when $V_{\mathrm{rf}}=0$, the ratio is actually calculated as the weighted average $\sum V_{\mathrm{sc}} V_{\mathrm{rf}} / \sum V_{\mathrm{rf}}^{2}$.

Transverse tune shifts are calculated using the Laslett tune shift formula without boundary terms:

$$
\Delta Q_{x, y}=-\frac{q^{2}}{4 \pi \epsilon_{0} m c^{2}} \frac{N}{2 \pi B \beta \gamma^{2} \epsilon_{x, y}^{\mathrm{n}, \mathrm{rms}}} \frac{\bar{\sigma}_{x, y}}{\bar{\sigma}_{x}+\bar{\sigma}_{y}},
$$

where the last term only requires the average beam aspect ratio $\bar{\sigma}_{x} / \bar{\sigma}_{y}$, which is 1 for ISIS and $\bar{\sigma}_{u} / \bar{\sigma}_{v}=0.554$ for the $12 \mathrm{GeV}$ VFFAG. There is a slight inconsistency with the transverse simulations that used a waterbag with $\epsilon_{x, y}^{\mathrm{rms}, 800 \mathrm{MeV}}=25 \mathrm{~mm}$ mrad whereas the $1 \mathrm{D}$ simulations assumed the value $30 \mathrm{~mm}$ mrad observed in the machine.

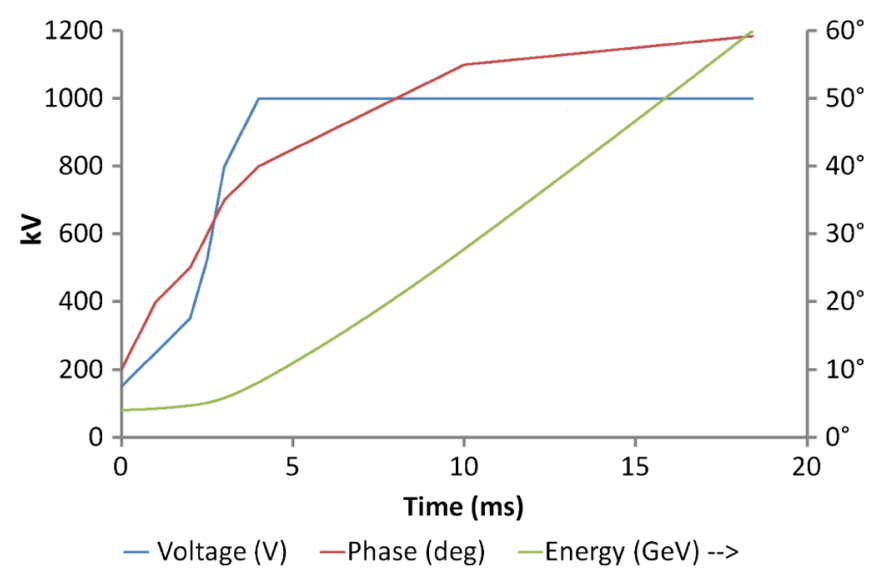

FIG. 15. Radio frequency acceleration program for the $12 \mathrm{GeV}$ VFFAG.

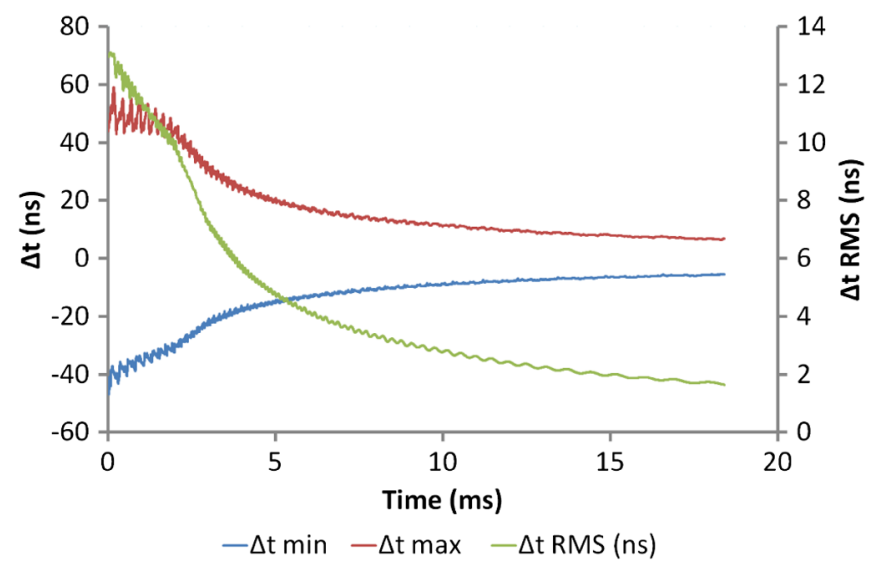

FIG. 16. Bunch duration in the $12 \mathrm{GeV}$ VFFAG simulation, reducing to a final value of $\Delta t_{\mathrm{rms}}=1.64 \mathrm{~ns}$.

\section{B. VFFAG rf system}

The VFFAG transfers the two bunches from ISIS into the 1 st and 3rd buckets of a frequency-doubled rf system given in Table II. Because of this doubling, the bunch initially fills much of the rf bucket, meaning only low acceleration

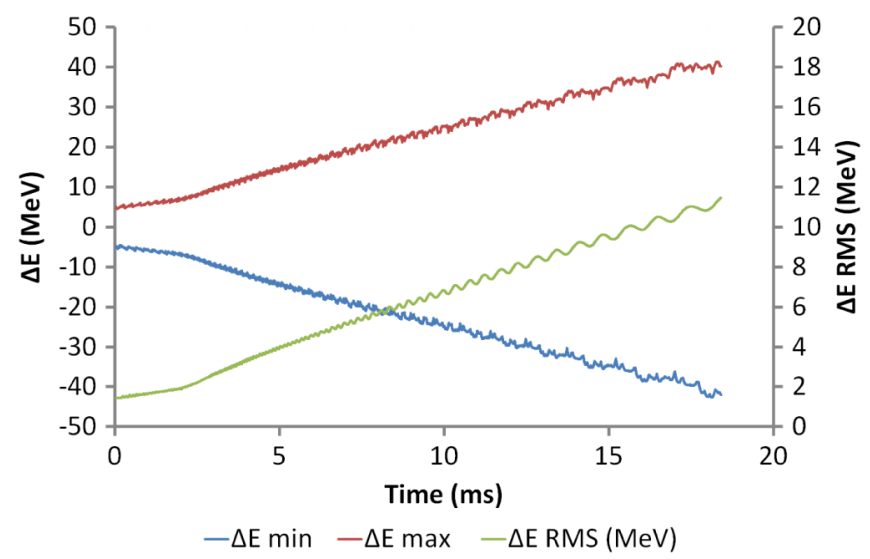

FIG. 17. Increase of bunch energy spread in the $12 \mathrm{GeV}$ VFFAG simulation to $\pm 41.1 \mathrm{MeV}$.

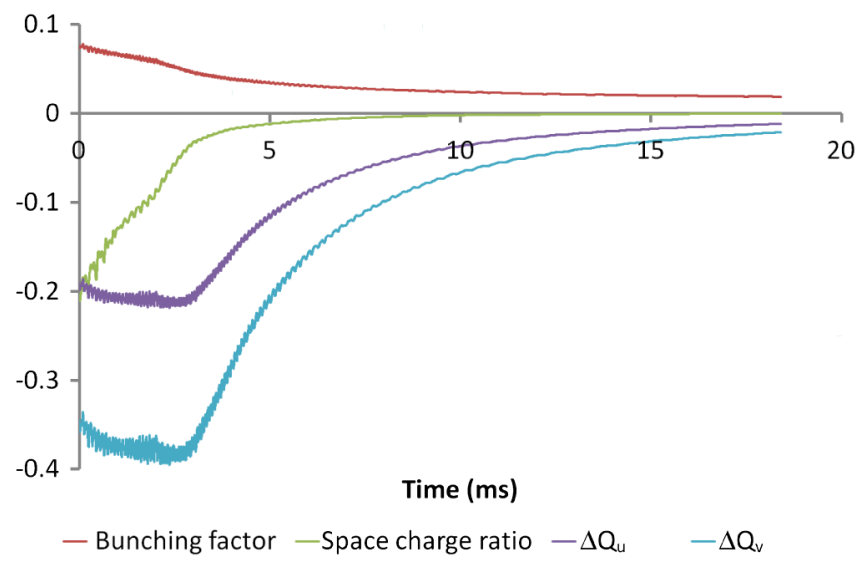

FIG. 18. Bunching factor and intensity-dependent effects in the $12 \mathrm{GeV}$ VFFAG simulation. 
TABLE III. Intensity-dependent parameters for the ISIS single harmonic and $12 \mathrm{GeV}$ VFFAG simulations run in series, for different numbers of protons injected into ISIS.

\begin{tabular}{lccc}
\hline \hline ISIS protons in & $2.50 \times 10^{13}$ & $2.75 \times 10^{13}$ & $3.00 \times 10^{13}$ \\
\hline ISIS $\mu \mathrm{A}$ in & 200.3 & 220.3 & 240.3 \\
ISIS transmission & $90.54 \%$ & $87.95 \%$ & $85.98 \%$ \\
ISIS protons out & $2.26 \times 10^{13}$ & $2.42 \times 10^{13}$ & $2.58 \times 10^{13}$ \\
ISIS $\mu \mathrm{A}$ out & 181.3 & 193.7 & 206.6 \\
ISIS power (kW) & 145 & 155 & 165 \\
VFFAG transmission & $100 \%$ & & \\
VFFAG power (MW) & 2.18 & 2.32 & 2.48 \\
ISIS peak intensities & & & \\
Bunching factor & 0.154 & 0.150 & 0.151 \\
Space charge ratio & -0.301 & -0.305 & -0.311 \\
$\Delta Q_{x, y}$ & -0.499 & -0.544 & -0.580 \\
$V F F A G$ peak intensities & & & \\
Bunching factor & 0.0188 & 0.0190 & 0.0190 \\
Space charge ratio & -0.211 & -0.257 & -0.278 \\
$\Delta Q_{u}$ & -0.219 & -0.240 & -0.254 \\
$\Delta Q_{v}$ & -0.395 & -0.434 & -0.458 \\
\hline \hline
\end{tabular}

phases are possible early in the cycle (Fig. 15). Later on, the bunch shrinks in time spread and increases in energy spread (Figs. 16 and 17), allowing faster acceleration.

In theory the bunch could be adiabatically compressed via an increase in rf voltage early in the cycle, followed by high-phase acceleration. However, as Fig. 18 shows, at low energies of $0.8-1.1 \mathrm{GeV}$ (the first $3 \mathrm{~ms}$ ) the tune shift in the $v$ plane is high and would exceed the half integer limit if full bunch compression was attempted near injection energy, hence the period of low-phase acceleration.

Table III shows the result of increasing the ISIS linac pulse length by $10 \%$ or $20 \%$. Worst-case intensity parameters are shown, with tune shifts in the VFFAG remaining below those of ISIS even with $14 \%$ more current. The large space charge tune shifts of $\sim-0.5$ are observed in ISIS during normal operation [16] and comparable to those in the CERN PS Booster [17]. Note that in reality ISIS now uses a more optimized rf program or its 2 nd harmonic system, giving lower losses and bunching factors.

\section{Suitability for muon production}

Note that in Fig. 16, the output rms bunch length is small enough to be used for a neutrino factory proton driver [18]. Some of the $1.59 \mathrm{~ms}$ spare time for $\mathrm{rf}$ reset at the end of the cycle could be used to produce an energy flat top so the two bunches can be extracted with a delay between.

\section{FUTURE DEVELOPMENT AND APPLICATIONS}

VFFAGs are promising candidates for a number of current accelerator needs, this section explores why and what further work there is to be done.

\section{A. Improving the proton driver design}

The proton driver rings presented in this paper show that in principle a VFFAG can have the right parameters for an ISIS upgrade. However, the lattice needs to be improved to make the machine more practical: although there is enough horizontal drift space for the proposed rf at $25 \%$ packing factor [11], the diagonal shape between the magnets makes it difficult to fit in a slotted rf cavity that spans the whole energy range. The remedy being considered is to make a racetrack-style ring out of two different lattices: an edgefocused lattice, like the current one but with shorter drifts, designed for maximum bending; and an insertion designed for long drifts but with no bending or edge angles. The insertion could contain rf, collimators, injection, and extraction equipment. This design approach has been successfully applied by Mori [19] for scaling FFAGs. Provided the $k$ of the two VFFAG sections is the same, there is no disagreement in closed orbit between them and the matching will be mostly concerned with reducing $\beta$ ripple.

\section{B. Isochronous VFFAGs for high average power}

Introducing some horizontal orbit excursion into a VFFAG varies the orbit circumference and allows it to be exactly proportional to beam velocity, making the machine isochronous. This is easier than in a horizontal orbit excursion FFAG (or a cyclotron) because the orbits do not have to converge towards a single location at the circumference corresponding to $v / c=1$. Such convergence would require magnetic gradients that increase faster than the beam momentum and eventually cause overfocusing, whereas vertical orbit motion can control the field gradient and even keep the normalized focusing strength constant.

The orbit excursion follows the path of increasing upward dipole field (where $B_{y}>0$ but $B_{x}=0$ ). When this path is at an angle $\theta$ to the horizontal in the $(x, y)$ plane, the quadrupole focusing associated with its gradient is at an angle $\theta / 2$ to a normal quadrupole. Horizontal FFAGs correspond to $\theta=0$ and pure VFFAGs to $\theta=90^{\circ}$ with the quadrupole then rotated by $\theta / 2=45^{\circ}$. For an isochronous VFFAG that is not ultrarelativistic, these angles will vary as the beam accelerates, tending towards $\theta=90^{\circ}$ as $v \rightarrow c$.

Isochronous machines may use a fixed-frequency rf system, which generally has a higher gradient than variablefrequency rf. If the orbit turns are also separated at injection and extraction, the VFFAG can operate in cw mode like a cyclotron. Figure 11 shows that the $2.8 \mathrm{~A}, 800 \mathrm{MeV}$ proton driver beam would require $8 \mathrm{~cm}$ to separate it from an adjacent turn if it were in an isochronous machine. Using the scaling beam size $\propto \sqrt{\epsilon}=\sqrt{\epsilon^{\mathrm{n}} / \beta \gamma}$ and $\epsilon^{\mathrm{n}} \propto$ current to keep the space charge tune shift constant, the turn separation needed for a $12 \mathrm{GeV}$ proton beam of $2.2 \mathrm{~mA}$ (the current of the PSI cyclotron) is $0.76 \mathrm{~mm}$. This is evaluated at the top energy because the turns are closest there relative 
to beam size, as shown in Fig. 1. The orbit motion satisfies $\mathrm{d} E / \mathrm{d} y=k \beta^{2} \gamma m c^{2}$, which for the $k=2.2 \mathrm{~m}^{-1}$ used in the $12 \mathrm{GeV}$ proton ring gives $28.3 \mathrm{MeV} / \mathrm{mm}$ at $12 \mathrm{GeV}$, so $\Delta E=21.4 \mathrm{MeV} /$ turn is required to separate turns. This compares to the $3 \mathrm{MeV} /$ turn used in the PSI cyclotron, which has an order of magnitude smaller circumference. Thus isochronous VFFAGs could potentially have the same beam current as a cyclotron but a much higher (relativistic) extraction energy, yielding an average beam power of 26.4 MW in this case. Such machines may be of interest for nuclear waste transmutation.

\section{Complete isochronism in strong focusing rings}

For rings dominated by strong focusing (i.e. large rings very unlike a cyclotron), it is possible to find an orbit excursion that is isochronous and fixed-tune analytically. As the VFFAG is also fixed field, this satisfies all of Teichmann's complete isochronism conditions [8].

For isochronism, the mean orbit radius must be $r=\beta R$ for some $R$ and for the orbit to close, $\left\langle B_{y}\right\rangle=p / q r=$ $m \beta \gamma c /(q \beta R)=\gamma B_{0}$ where $B_{0}=m c / q R$. This is familiar as the field relation for an isochronous cyclotron. Assume $B_{y}$ varies in sectors around the machine but always retains the scaling with $\gamma$. The normalized focusing strength of a magnet is $B^{\prime} l / p$ where $l=r \Theta$ is the sector magnet length at momentum $p$ and $B^{\prime}$ is the magnitude of the quadrupole gradient, regardless of quadrupole orientation:

$$
\frac{B^{\prime} l}{p}=\frac{B^{\prime} r \Theta}{m \beta \gamma c}=\frac{B^{\prime} \beta R \Theta}{m \beta \gamma c}=\frac{B^{\prime}}{\gamma} \frac{R \Theta}{m c} \propto \frac{B^{\prime}}{\gamma},
$$

so $B^{\prime}$ must be proportional to $\gamma$ to keep normalized focusing (and hence machine tunes) constant. However, this means $\mathrm{d} B_{y} / \mathrm{d} s \propto \gamma \propto B_{y}$, where $s$ is path length in $(x, y)$ along the orbit excursion curve. Thus $B_{y}=B_{0} \mathrm{e}^{s / S}$ for an appropriate choice of origin for $s$ and $S=1 / k$ is the scaling length. In other words, the dipole field must increase exponentially along the orbit excursion curve to retain scaling optics.

The actual shape of the orbit excursion is found via the relation between $\beta$ and $\gamma$ :

$$
r=\beta R=R \sqrt{1-\gamma^{-2}}=R \sqrt{1-\mathrm{e}^{-2 s / S}}
$$

relates arclength to $r$. It also provides a lower bound to the energy used in such a machine because after some manipulation,

$$
\frac{\mathrm{d} r}{\mathrm{~d} s}=\frac{R}{S} \frac{1}{\beta \gamma^{2}}
$$

but $\mathrm{d} r / \mathrm{d} s \leq 1$ because $s$ is path length, therefore

$$
\beta \gamma^{2} \geq \frac{R}{S}
$$

in a strong-focusing-dominated, isochronous VFFAG with scaling optics. The equality $\beta \gamma^{2}=R / S$ occurs at the minimum energy when the orbit excursion is horizontal.
$S$ dictates the separation of the orbits, so increasing $S$ allows the acceleration to start from lower energies but increases the size of the magnets in the machine.

It should be noted that in smaller machines, the weak focusing in $x$ from the rotation around the ring is significant and in fact dominant in many cyclotrons. Cyclotrons clearly work to much lower energies, so an analysis including weak focusing may keep the eigentunes constant to lower energies if the ring tunes are low.

\section{Electron VFFAGs and eRHIC}

The lower bound on energy for a scaling isochronous VFFAG gets easier to satisfy the lower the mass of the particle being accelerated. For electron machines in the $\mathrm{GeV}$ scale, the difference from the speed of light becomes negligible and a scaling VFFAG with a purely vertical orbit excursion can be used.

Some recent ongoing work [20] has investigated the use of such a VFFAG for the proposed eRHIC project where an electron beam is recirculated through a fixed-frequency linac several times in the RHIC tunnel and then decelerated in the same machine, out of phase, for energy recovery. The energy range in one example was $1.2-10 \mathrm{GeV}$ over eight linac passes. $k=100 \mathrm{~m}^{-1}$ was used, meaning the turns fitted within an orbit excursion of $21.2 \mathrm{~mm}$. Here a single VFFAG transfer line replaced either a cascade of two nonscaling FFAGs, or several stacked ring transfer lines, due to its acceptance of a high momentum range. Energy loss from synchrotron radiation is higher than in a comparable ring without reverse magnet bends but is still manageable.

\section{Hadron therapy}

Finally, VFFAGs may be of interest for medical proton and ion therapy machines. The PAMELA [21] study has investigated conventional FFAGs for these applications because of their advantages of compactness and rapid cycling over synchrotrons. Compactness comes from their use of superconducting bending magnets and allows the machine to fit more easily in a hospital, while rapid cycling comes from not having to ramp the magnets and allows radiation doses to be applied with a finer resolution within the patient.

Since the beam in these applications is transversely small, the VFFAG style magnet (see Sec. II B) may reduce the magnet volume down to a narrow slot, further reducing the amount of material and power used in the machine, while still preserving the other advantages of FFAGs listed above.

[1] K. R. Symon, D. W. Kerst, L. W. Jones, L. J. Laslett, and K. M. Terwilliger, Phys. Rev. 103, 1837 (1956).

[2] J.L. Powell, Eq. (1) in MURA report 80, available from http://cdsweb.cern.ch/record/1052338/files/cer002709483.pdf (1955). 
[3] S. J. Brooks, in Proceedings of the 46th ICFA Advanced Beam Dynamics Workshop on High-Intensity and HighBrightness Hadron Beams (PSI, Viligen, Switzerland, 2010), p. 96.

[4] K. R. Symon, MURA note 043, 1954.

[5] D. J. Kelliher et al. (RAL Collaboration) (private communication).

[6] Reported by T. Ohkawa at the meeting of the Physical Society of Japan, 1955 (unpublished).

[7] G. Leleux, J. Proy, and M. Salvat, Rapport OC 70, Service de Physique Appliquee Section d'Optique Corpusculaire (1959).

[8] J. Teichmann, Sov. J. At. Energ. 12, 507 (1963).

[9] S. J. Brooks (unpublished) [http://stephenbrooks.org/ral/ report/2011-6/skewVFFAGfringes.pdf].

[10] B. Boardman, Rutherford Appleton Laboratory Technical Report No. RL-82-006, 1982.

[11] S.J. Brooks, in Proceedings of the 23rd Particle Accelerator Conference, Vancouver, Canada, 2009 (Ref. [22]), p. 4357.

[12] R. Baartman, TRIUMF Design Note 1992-TRI-DN-K206 [http://lin12.triumf.ca/text/design_notes/k206/k206h.pdf].

[13] S. J. Brooks (unpublished) [http://stephenbrooks.org/ral/ report/2012-6/abeamupipe.pdf], Sec. 2.6.2.

[14] C. R. Prior et al. (RAL Collaboration) (private communication).
[15] A. Hofmann and F. Pedersen, in Proceedings of PAC'79 [IEEE Trans. Nucl. Sci. 26, 3527 (1979)].

[16] B. G. Pine and C. M. Warsop, in Proceedings of the 52nd ICFA Advanced Beam Dynamics Workshop on HighIntensity and High-Brightness Hadron Beams (2012), p. 206.

[17] B. Mikulec, A. Findlay, V. Raginel, G. Rumolo, and G. Sterbini, in Proceedings of the 52nd ICFA Advanced Beam Dynamics Workshop on High-Intensity and HighBrightness Hadron Beams (Ref. [16]), p. 178.

[18] S. Choubey et al. (The IDS-NF collaboration), Report No. RAL-TR-2011-018, Table V, p. 55.

[19] J. B. Lagrange, T. Planche, E. Yamakawa, Y. Ishi, Y. Kuriyama, Y. Mori, K. Okabe, and T. Uesugi, in Proceedings of the IPAC'10 Conference, Kyoto, Japan (ICR, Kyoto, 2010), p. 4503.

[20] D. Trbojevic et al. (BNL Collaboration) (private communication).

[21] K. Peach et al., in Proceedings of the 23rd Particle Accelerator Conference, Vancouver, Canada, 2009 (Ref. [22]), p. 3142.

[22] H. Witte, T. Yokoi, S. Sheehy, J. Cobb, and K. Peach, in Proceedings of the 23rd Particle Accelerator Conference, Vancouver, Canada, 2009 (IEEE, Piscataway, NJ, 2009), p. 301. 